\title{
Distinct contributions of the basolateral amygdala and the medial prefrontal cortex to learning and relearning extinction of context conditioned fear
}

\author{
Vincent Laurent and R. Frederick Westbrook ${ }^{1}$ \\ School of Psychology, The University of New South Wales, Sydney, New South Wales 2052, Australia
}

\begin{abstract}
We studied the roles of the basolateral amygdala (BLA) and the medial prefrontal cortex (mPFC) in learning and relearning to inhibit context conditioned fear (freezing) in extinction. In Experiment 1, pre-extinction BLA infusion of the NMDA receptor (NMDAr) antagonist, ifenprodil, impaired the development and retention of inhibition but post-extinction infusion spared retention. Pre-extinction infusion of the $\mathrm{GABA}_{\mathrm{A}}$ agonist, muscimol, depressed freezing and impaired retention as did post-extinction infusion. In Experiment 2, pre-extinction mPFC infusion of ifenprodil spared the development of inhibition whereas muscimol depressed freezing. Both impaired retention when infused pre- or post-extinction. Thus, the development of inhibition involves NMDAr activation in the BLA, whereas its consolidation involves both NMDAr activation in the mPFC and NMDAr-independent mechanisms in the BLA. In Experiment 3, BLA infusion of ifenprodil impaired relearning and retention of inhibition when infused before but did not impair retention when infused after re-extinction. BLA infusion of muscimol depressed freezing but did not impair retention when infused before or after re-extinction. In Experiment 4, mPFC infusion of ifenprodil impaired relearning when infused before re-extinction, whereas muscimol depressed responses. Both drugs impaired retention when infused into the mPFC before or after re-extinction. Thus, relearning to inhibit fear responses involves NMDAr activation in both the BLA and mPFC and consolidation of the inhibitory memory involves NMDAr activation in the mPFC. However, relearning and consolidation occur in the absence of neuronal activity within the BLA. We propose that NMDAr in the mPFC supports relearning inhibition when the BLA is inactivated.
\end{abstract}

Extinction of Pavlovian conditioned fear responses occurs when the association between a conditioned place or stimulus (CS) and an aversive unconditioned stimulus (US) is broken by exposures to the CS in the absence of the US. The fear responses elicited by the CS decline across CS alone exposures and eventually cease to occur. Fear responding to the CS is said to be extinguished. Theories which identified learning with a single construct, e.g., associative strength (e.g., Rescorla and Wagner 1972) or connection weight (Rumelhart and McClelland 1986), explained extinction as erasure of this strength or reversal of the connection weights between the CS and US. However, there is now considerable evidence that much, if not all, of the original CS-US association survives extinction in spite of the fact that the CS fails to elicit fear responses. For instance, fear responses recover spontaneously with the passage of time since extinction; they are renewed when the CS is tested outside the context where extinction occurred; and are reinstated when the extinguished CS is tested in or shortly after exposure to a dangerous context. These fear restoration phenomena have led to the view that extinction is an example of occasion setting whereby the various cues (temporal, physical, and internal) present across extinction come to activate inhibitory associations which prevent retrieval of the CS-US association or its expression in fear responses (Bouton et al. 2006). Just as the CS-US association survives extinction, the inhibition acquired in extinction survives reconditioning. For instance, an extinguished CS subjected to further fear conditioning continues to show extinction phenomena such as spontaneous recovery and renewal (Bouton and Swartzentruber 1989; Res-

\footnotetext{
1Corresponding author.
}

E-mail f.westbrook@unsw.edu.au; fax 61-92-03853044.

Article is online at http://www.learnmem.org/cgi/doi/10.1101//m.1080108. corla 2001). This implies that the learning which produced these phenomena remains intact in spite of the fact that the CS elicits fear responding.

The amygdala is critical for acquisition and consolidation of extinction learning. Acquisition is impaired by blockade of NMDA receptors (NMDAr), metabotropic glutamate receptors, or mitogen-activated protein kinase (MAPk) activity in the basolateral amygdala (BLA) (Herry et al. 2006; Kim et al. 2007; SotresBayon et al. 2007). Consolidation involves activation in the BLA of the phosphoinositide-3 kinase pathway, synthesis of new protein, and the expression of immediate early genes (Lin et al. 2003; Herry and Mons 2004). The medial prefrontal cortex (mPFC) is also required for consolidation and may serve to activate mechanisms in the amygdala that suppress fear responses. Blockade of NMDAr, MAPk, or protein synthesis in the mPFC does not impair development of inhibition in extinction but does impair retention of the inhibitory memory (Santini et al. 2004; Hugues et al. 2006; Burgos-Robles et al. 2007). Stimulation of the mPFC inhibits activity of neurons in the central nucleus of the amygdala (CeA) that mediate fear responses (Quirk et al. 2003), and neurons in the MPFC exhibit enhanced firing to an extinguished CS (Milad and Quirk 2002). These findings have led to the suggestion that projections from the MPFC activate a network of GABAergic cells located between the BLA and CeA, leading to a suppression of fear responses (Quirk and Mueller 2008).

The BLA is critical for learning to inhibit fear responses in extinction. However, once this inhibition has been acquired, the BLA is not required for learning anew this inhibition. Disruption of neuronal activity in the BLA by infusion of the GABA $\mathrm{A}_{\mathrm{A}}$ agonist, muscimol (Laurent et al. 2008), or the anesthetic, bupivacaine (Kim and Richardson 2008), before extinction resulted in fear responses when rats were subsequently assessed for retention. 
However, rats subjected to two cycles of fear conditioning and fear extinction and infused with muscimol or bupivacaine before the second extinction did not show fear responses on the subsequent retention test. This suggests that compensatory mechanisms allow rats to relearn extinction when the BLA is inactivated. As noted previously, conditioning an extinguished CS restores fear responses but leaves intact the inhibitory learning produced by extinction. Therefore, these compensatory mechanisms may consist in reactivating and strengthening the original inhibitory memory that is stored and expressed by other structures than the BLA. One candidate is the MPFC. However, its role in relearning inhibition is unknown. The present experiments subjected rats to a single cycle of conditioning and extinction or two such cycles to study the roles played by the BLA and the mPFC in learning and relearning extinction. In Experiment 1, ifenprodil, an antagonist for NMDAr containing the NR2B subunit, or muscimol was infused into the BLA before or after initial extinction. In Experiment 2, ifenprodil or muscimol was infused into the mPFC before or after initial extinction. In Experiment 3, ifenprodil or muscimol was infused into the BLA before or after the second extinction, and, in Experiment 4, ifenprodil or muscimol was infused into the mPFC before or after the second extinction.

\section{Results}

\section{Experiment 1: The role of the BLA in the learning produced by extinction}

There is evidence that the learning produced by extinction requires activation of NMDAr in the BLA (Falls et al. 1992; Lee and Kim 1998; Sotres-Bayon et al. 2007). However, it is unclear whether activation of these receptors is also required for the consolidation of this learning. For instance, systemic injection of an NMDAr antagonist either before or after extinction resulted in fear responses on a subsequent retention test, indicating impairment in the formation of the inhibitory memory (Santini et al. 2001; Goosens and Maren 2004). A BLA infusion of ifenprodil, a selective antagonist of NMDAr containing the NR2B subunit, also impaired formation of the inhibitory memory when administered before extinction (Sotres-Bayon et al. 2007), but the effects of NMDAr blockade in the BLA following extinction are unknown. Therefore, Experiment 1 provided a further study of the role played by NMDAr activation within the BLA in the acquisition and the consolidation of the learning produced by extinction. This was achieved by infusing ifenprodil before or after a first extinction session. Since other mechanisms, as well as NMDAr activation may be involved, we also assessed the effects of inactivating the BLA by infusing the $\mathrm{GABA}_{\mathrm{A}}$ agonist muscimol either before or after extinction. Rats were submitted to a conditioning episode on days 1 and 3 , and to an extinction session on day 4 (Fig. 2A, see below). They received this extinction session under vehicle (Groups VEH/VEH, VEH/IFEN, and VEH/MUSC), ifenprodil (Group IFEN/VEH), or muscimol (Group MUSC/VEH). Immediately after extinction, some of the rats that had received vehicle now received ifenprodil (Group VEH/IFEN) or muscimol (Group VEH/MUSC) whereas all the remaining rats received vehicle for their first (Groups IFEN/VEH and MUSC/VEH) or second time (Group VEH/VEH). Finally, all rats received a drug-free retention test on day 5.

Figure $1 \mathrm{~A}$ shows the location of microinjection tips. A total of six rats were excluded because of incorrect cannula placement. This yielded the following group sizes: VEH/VEH $(n=6)$, IFEN/ VEH $(n=8)$, VEH/IFEN $(n=7)$, MUSC/VEH $(n=7)$, and VEH/ MUSC $(n=6)$.

The context conditioning episodes were successful. On day
A

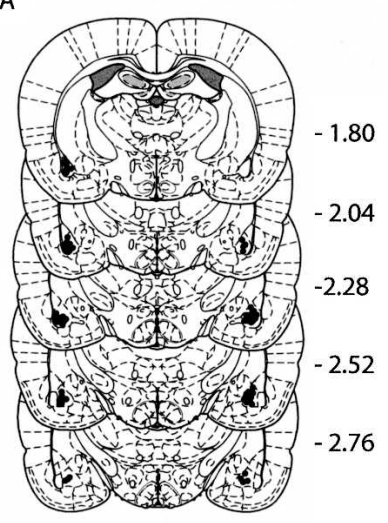

B

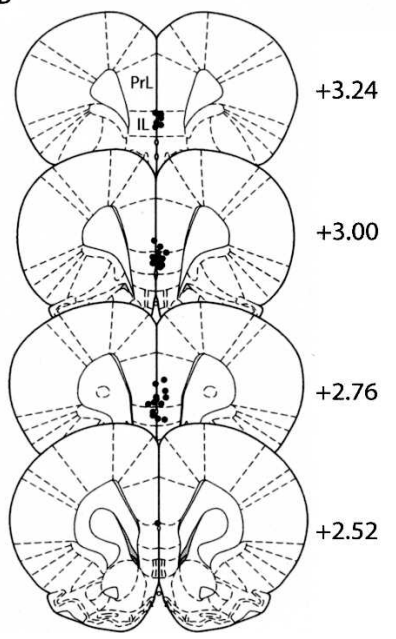

C

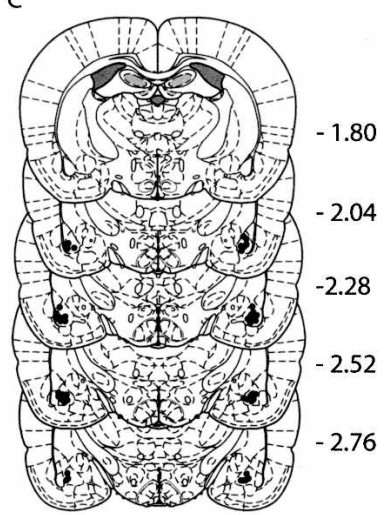

Figure 1. Location of microinjection cannulas within the BLA or the $\mathrm{mPFC}$ for all rats included in the final analysis of Experiments $1(A), 2(B)$, $3(C)$, and $4(D)$. Distances on the atlas templates (adapted from Paxinos and Watson 2007, reprinted with permission from Elsevier ( 2005) are indicated in millimeters from bregma.

4 (Fig. 2B), rats that received extinction under vehicle (Groups VEH/VEH, VEH/IFEN, and VEH/MUSC) showed substantial levels of freezing that gradually declined. BLA infusion of ifenprodil impaired the short-term inhibition of fear responses. Rats that received extinction under ifenprodil (Group IFEN/VEH) spent significantly more time freezing than rats treated with vehicle (Groups VEH/VEH, VEH/IFEN, and VEH/MUSC; $F_{(1,29)}=19.187$, $P<0.05)$. In contrast, rats infused with muscimol before extinction (Group MUSC/VEH) spent significantly less time freezing than vehicle-treated rats $\left(F_{(1,29)}=10.725, P<0.05\right)$. Overall, the levels of freezing declined across the extinction session $\left(F_{(1,29)}=205.637, P<0.05\right)$.

The test data on day 5 (Fig. 2C) showed that activation of NMDAr containing the NR2B subunit in the BLA is required for acquisition but not consolidation of extinction learning. Rats infused with ifenprodil before extinction (Group IFEN/VEH) froze significantly more than control rats infused with vehicle (Group VEH/VEH; $F_{(1,29)}=16.953, P<0.05$ ). In contrast, there was no significant difference between rats infused with ifenprodil after extinction (Group VEH/IFEN) and control rats (Group VEH/ VEH; $\left.F_{(1,29)}=0.236, P>0.05\right)$. Inactivation of the BLA prior to extinction impaired the long-term inhibition of fear. Rats treated with muscimol (Group MUSC/VEH) froze significantly more 


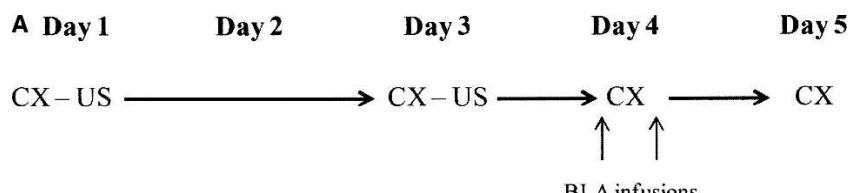

B

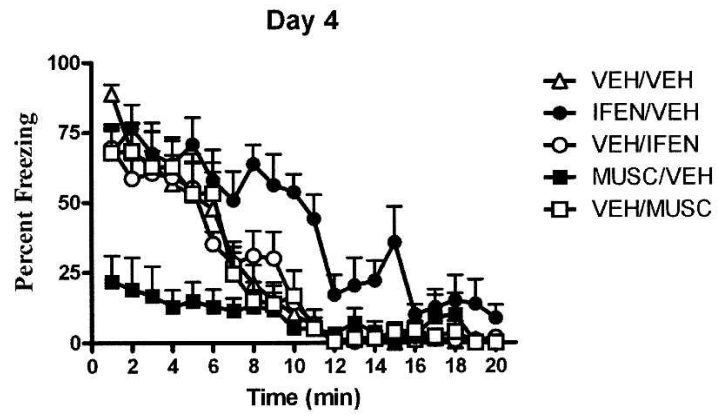

C

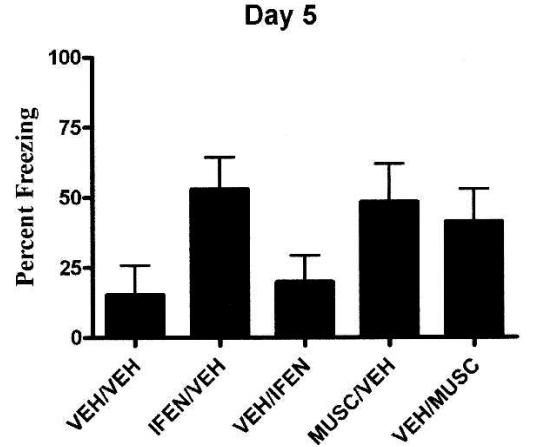

Figure 2. The role of the BLA in the learning produced by extinction. (A) Description of the behavioral procedures used in Experiment 1 (CX US: shocked exposure to the chambers; $C X$ : non-shocked exposure to the chambers). (B) All illustrations show the mean and SEM levels of freezing. Rats infused in the BLA with vehicle before extinction (Groups VEH/VEH, VEH/IFEN, and VEH/MUSC) decreased their freezing responses across the session. BLA infusion of ifenprodil impaired the decrease of fear responses in extinction (Group IFEN/VEH) whereas muscimol suppressed these responses (Group MUSC/VEH). (C) The retention test showed that ifenprodil disrupted acquisition (Group IFEN/VEH) but not consolidation of extinction (Group VEH/IFEN). In contrast, BLA infusion of muscimol disrupted both (Groups MUSC/VEH and VEH/MUSC). Freezing declined across the retention test in this and the other experiments in a manner consistent with the overall differences between the groups (data not shown).

than control rats on test (Group VEH/VEH; $F_{(1,29)}=12.535$, $P<0.05)$. Moreover, inactivation of the BLA by infusion of muscimol immediately after extinction disrupted consolidation of extinction learning as rats in Group VEH/MUSC froze significantly more than control rats (Group VEH/VEH; $F_{(1,29)}=7.139$, $P<0.05)$.

Experiment 2: The role of the mPFC in the learning produced by extinction

The previous experiment found that activation of NMDAr containing the NR2B subunit in the BLA is used to encode the inhibition produced by extinction but is not used for its consolida- tion. However, NMDAr activation in other brain regions may be necessary for consolidation (Santini et al. 2001). One of these is the mPFC. Infusion of an NMDAr antagonist before or after extinction impairs long-term inhibition of fear responses (BurgosRobles et al. 2007). The present experiment therefore examined the effects of infusing ifenprodil into the mPFC before or after extinction. As in Experiment 1, we also examined the effects of inactivating the $\mathrm{mPFC}$ with muscimol. Thus, rats received a conditioning episode on days 1 and 3 (Fig. 3A). On day 4, rats received an mPFC infusion of vehicle (Groups VEH/VEH, VEH/ IFEN, and VEH/MUSC), ifenprodil (Group IFEN/VEH), or muscimol (Group MUSC/VEH) and were subjected to an extinction session. Immediately after this session, they received a second infusion into the mPFC. Some of the rats that had received vehicle now received ifenprodil (Group VEH/IFEN) or muscimol

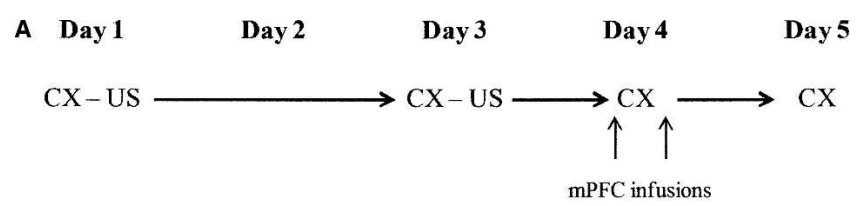

B

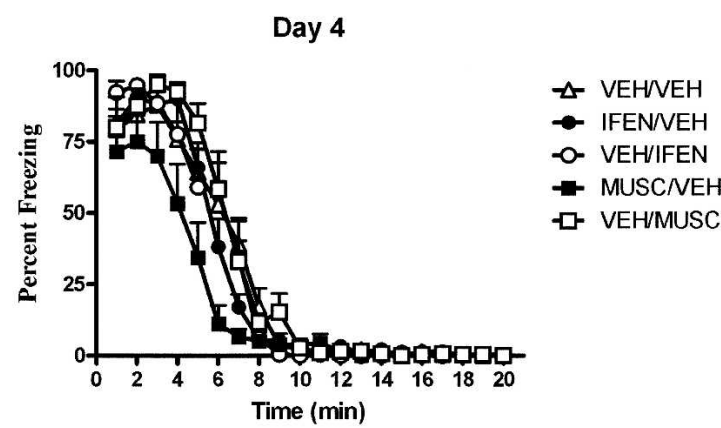

C

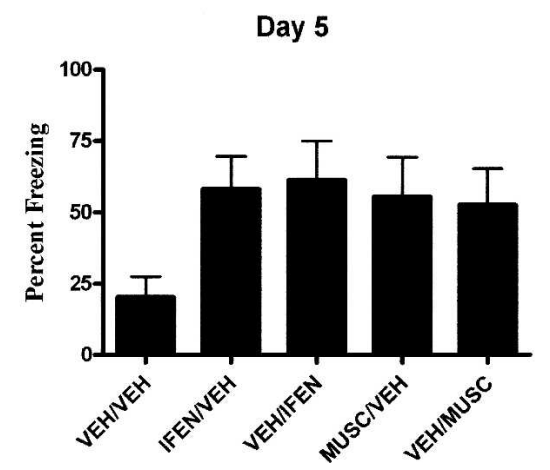

Figure 3. The role of the $\mathrm{MPFC}$ in the learning produced by extinction. (A) Description of the behavioral procedures used in Experiment 2 (CX US: shocked exposure to the chambers; CX: non-shocked exposure to the chambers). (B) All illustrations show the mean and SEM levels of freezing. Rats that performed extinction under vehicle or ifenprodil (Groups VEH/VEH, VEH/IFEN, VEH/MUSC, and IFEN/VEH) decreased their freezing responses across the session. mPFC infusion of muscimol depressed fear responses (Group MUSC/VEH). (C) The retention test showed that ifenprodil and muscimol disrupted consolidation but not acquisition of initial extinction (Groups IFEN/VEH, VEH/IFEN, MUSC/VEH, and VEH/MUSC). 
(Group VEH/MUSC) whereas all the remaining rats received vehicle for their first (Groups IFEN/VEH and MUSC/VEH) or second time (Group VEH/VEH). Finally, retention of the inhibitory memory was assessed by a drug-free test on day 5 .

Figure $1 \mathrm{~B}$ shows the location of microinjection tips. A total of seven rats were excluded because of incorrect cannula placement. This yielded the following group sizes: VEH/VEH $(n=14)$, IFEN/VEH $(n=9), \mathrm{VEH} / \mathrm{IFEN}(n=7), \mathrm{MUSC} / \mathrm{VEH}(n=6)$, and VEH/MUSC $(n=10)$.

Context conditioning was successful. On day 4 (Fig. 3B), rats infused with vehicle and subjected to extinction (Groups VEH/ VEH, VEH/IFEN, and VEH/MUSC) displayed substantial levels of freezing that gradually declined. These levels were similar to those shown by rats extinguished under ifenprodil (Group IFEN/ VEH; $\left.F_{(1,41)}=0.523, P>0.05\right)$. In contrast, mPFC infusion of muscimol depressed fear responses. Rats that received muscimol prior to extinction (Group MUSC/VEH) froze significantly less than vehicle-treated rats (Groups VEH/VEH, VEH/IFEN, and VEH/MUSC; $\left.F_{(1,41)}=10.496, P<0.05\right)$. Overall, levels of freezing declined across the session $\left(F_{(1,41)}=766.811, P<0.05\right)$.

The test data on day 5 (Fig. 3C) showed that activation of NMDAr containing the NR2B subunit in the mPFC is necessary for consolidation of extinction learning. Rats that had been infused with ifenprodil after extinction (Group VEH/IFEN) froze significantly more than control rats (Group VEH/VEH; $\left.F_{(1,41)}=33.406, P<0.05\right)$. Ifenprodil also impaired the long-term inhibition of fear when infused prior to extinction (Group IFEN/ VEH; $\left.F_{(1,41)}=33.301, P<0.05\right)$. However, this effect is likely to reflect a disruption of consolidation as ifenprodil did not impair the inhibition of freezing across extinction. Consistent with this evidence that NMDAr activation in the MPFC is critical for consolidation, disruption of neuronal activity induced by muscimol before or after extinction impaired the long-term inhibition of fear responses. Rats in Groups MUSC/VEH and VEH/MUSC froze significantly more on the retention test than control rats (Group VEH/VEH; respectively: $F_{(1,41)}=22.058, P<0.05$ and $\left.F_{(1,41)}=26.040, P<0.05\right)$.

\section{Experiment 3: The role of the BLA in the learning produced by re-extinction}

Experiment 1 showed that activation of NMDAr in the BLA is required to learn but not to consolidate the inhibition produced by extinction. However, it also showed that consolidation does require neural activity in the BLA. The present experiment examined whether these conclusions also characterized relearning this inhibition. In Experiment 3, we submitted rats to a conditioning episode on days 1 and 3 and to extinction on days 2 and 4 (Fig. 4A). Rats received an infusion of ifenprodil or muscimol into the BLA either before (Groups IFEN/VEH and MUSC/VEH) or after the second extinction session (i.e., re-extinction) (Groups VEH/IFEN and VEH/MUSC). A control group of rats received vehicle twice (Group VEH/VEH). Retention of the inhibitory memory was assessed by a drug-free test on day 5 .

Figure 1C shows the location of microinjection tips. A total of nine rats were excluded because of incorrect cannula placement. This yielded the following group sizes: VEH/VEH $(n=6)$, IFEN/VEH $(n=6), \mathrm{VEH} / \mathrm{IFEN}(n=6), \mathrm{MUSC} / \mathrm{VEH}(n=7)$, and VEH/MUSC $(n=6)$.

Conditioning was successful on day 1 . All rats showed substantial levels of freezing when returned to the conditioning chambers for the first extinction session on day 2 (Fig. 4B). These levels gradually and significantly declined over the course of the session $\left(F_{(1,27)}=132.909, P<0.05\right)$. The second conditioning episode on day 3 restored extinguished fear responses as rats that received re-extinction under vehicle (Groups VEH/VEH, VEH/

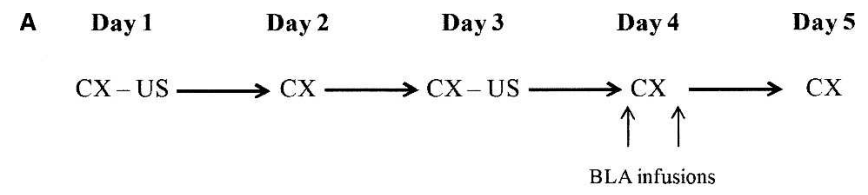

B

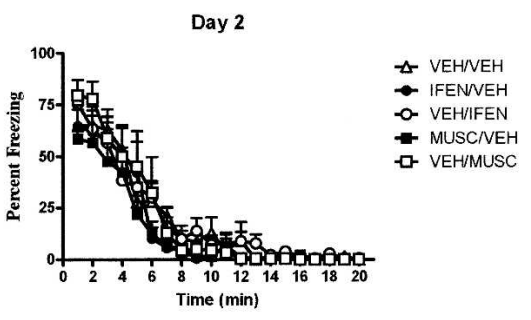

C

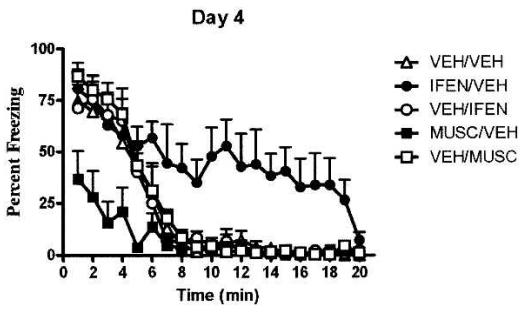

D

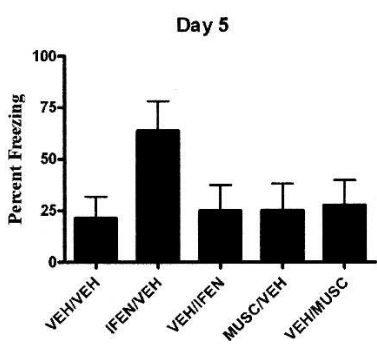

Figure 4. The role of the BLA in the learning produced by re-extinction. (A) Description of the behavioral procedures used in Experiment 3 (CX US: shocked exposure to the chambers; CX: non-shocked exposure to the chambers). (B) All illustrations show the mean and SEM levels of freezing. All rats decreased their freezing responses across initial extinction. (C) Rats infused in the BLA with vehicle before re-extinction (Groups VEH/VEH, VEH/IFEN, and VEH/MUSC) decreased their freezing responses across the session. BLA infusion of ifenprodil impaired the decrease of fear responses in re-extinction (Group IFEN/VEH) whereas muscimol suppressed those responses (Group MUSC/VEH). (D) The retention test showed that ifenprodil impaired acquisition (Group IFEN/VEH) but not consolidation (Group VEH/IFEN) of re-extinction. In contrast, BLA infusion of muscimol (Groups MUSC/VEH and VEH/MUSC) disrupted neither acquisition nor consolidation.

IFEN, and VEH/MUSC) displayed substantial levels of freezing on day 4 (Fig. 4C). As in the case of extinction (Experiment 1), a BLA infusion of ifenprodil impaired the inhibition of fear responses across re-extinction. Rats that had received ifenprodil prior to re-extinction froze significantly more than rats that had received vehicle (Groups VEH/VEH, VEH/IFEN, and VEH/MUSC; $\left.F_{(1,27)}=47.410, P<0.05\right)$. In contrast, these vehicle-treated rats froze significantly more than rats infused with muscimol (Group MUSC/VEH; $\left.F_{(1,27)}=13.742, P<0.05\right)$. Thus, inactivation of the BLA by muscimol depressed fear responses in re-extinction as it had depressed these responses in extinction (Experiment 1). Overall, levels of freezing gradually and significantly declined across the re-extinction session $\left(F_{(1,27)}=102.898, P<0.05\right)$.

The test data on day 5 (Fig. 4D) showed that activation of 
NMDAr containing the NR2B subunit is necessary for acquisition but not consolidation of re-extinction. Rats that had been infused with ifenprodil before re-extinction (Group IFEN/VEH) froze more than control rats (Group VEH/VEH; $F_{(1,27)}=16.953$, $P<0.05)$, whereas rats infused with ifenprodil after re-extinction (Group VEH/IFEN) displayed similar levels of freezing as control rats $\left(F_{(1,27)}=0.172, P>0.05\right)$. In contrast to its disruptive effects when administered before or after the initial extinction, muscimol spared relearning whether infused before or after the second extinction session. Rats treated with muscimol (Groups MUSC/ VEH and VEH/MUSC) exhibited low levels of freezing on the retention test, similar to those by control rats (Group VEH/VEH; respectively: $F_{(1,27)}=0.212, P>0.05$ and $\left.F_{(1,27)}=0.514, P>0.05\right)$. Thus, NMDAr activation in the BLA is critical for relearning to inhibit fear responses in extinction. However, inactivation of the BLA did not impair this relearning, suggesting that other brain regions compensate when the BLA is inactivated and allow rats to relearn inhibition (Laurent et al. 2008).

\section{Experiment 4: The role of the mPFC in the learning produced by re-extinction}

Experiments 1 and 3 have shown that learning and relearning to inhibit fear responses in extinction involve different substrates. For instance, BLA inactivation impaired the learning produced by initial extinction but spared relearning produced by the second extinction. The present experiment examined whether learning and relearning extinction also differs with respect to NMDAr-dependent plasticity and neuronal activity in the mPFC. Rats received a conditioning episode on day 1, extinction training on day 2, and a second conditioning episode on day 3 (Fig. $5 \mathrm{~A})$. On day 4 , rats were submitted to a re-extinction session following mPFC infusion of vehicle (Groups VEH/VEH, VEH/ IFEN, and VEH/MUSC), ifenprodil (Group IFEN/VEH), or muscimol (Group MUSC/VEH). A second infusion was given immediately after the session. Some of the rats that had received vehicle now received ifenprodil (Group VEH/IFEN) or muscimol (Group VEH/MUSC) whereas all the remaining rats received vehicle for their first (Groups IFEN/VEH and MUSC/VEH) or second time (Group VEH/VEH). Finally, all rats received a drug-free retention test on day 5 .

Figure 1D shows the location of microinjection tips. A total of twenty-two rats were excluded because of incorrect cannula placement. This yielded the following group sizes: VEH/VEH $(n=11)$, IFEN/VEH $(n=10)$, VEH/IFEN $(n=10)$, MUSC/VEH $(n=9)$, and VEH/MUSC $(n=9)$.

Context conditioning on day 1 was successful. All rats showed substantial levels of freezing when returned to the chambers for extinction on day 2 (Fig. 5B). Fear responses gradually declined in extinction $\left(F_{(1,44)}=368.430, P<0.05\right)$. These responses were restored by the second conditioning episode on day 3. On day 4 (Fig. 5C), rats that received re-extinction under vehicle (Groups VEH/VEH, VEH/IFEN, and VEH/MUSC) showed substantial levels of freezing that gradually declined. Rats infused with ifenprodil (Group IFEN/VEH) were impaired in acquiring this inhibition of fear responses across the re-extinction session; they froze significantly more than rats infused with vehicle (Groups VEH/VEH, VEH/IFEN, and VEH/MUSC; $F_{(1,44)}=9.929$, $P<0.05)$. This impairment in response inhibition across reextinction contrasts with the absence of any such effect across extinction (Experiment 2) but is similar to the impairments produced by infusion into the BLA (Experiments 1 and 3). Rats infused with muscimol showed significantly less freezing across re-extinction than vehicle-treated rats $\left(F_{(1,44)}=13.608, P<0.05\right)$. Overall, the levels of freezing declined across re-extinction $\left(F_{(1,44)}=1180.614, P<0.05\right)$.
A

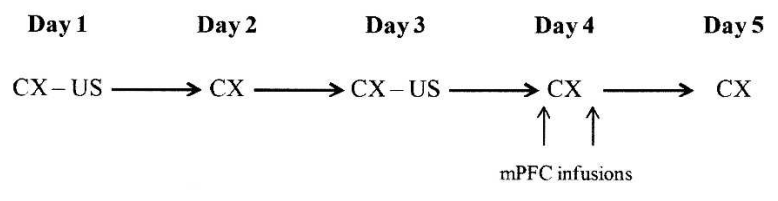

B

Day 2

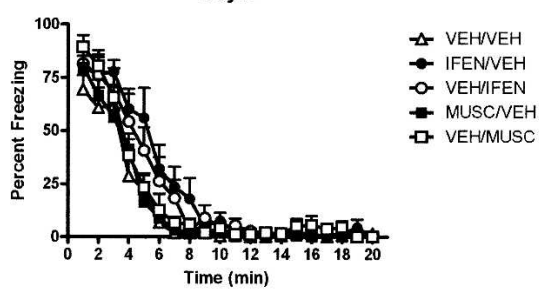

C

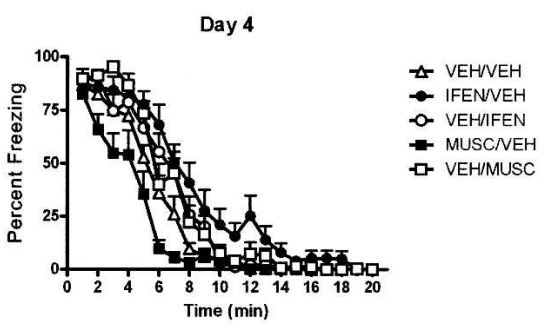

D

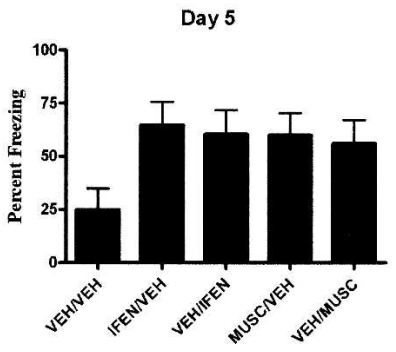

Figure 5. The role of the $\mathrm{mPFC}$ in the learning produced by reextinction. (A) Description of the behavioral procedures used in Experiment 4 ( $C X$ - US: shocked exposure to the chambers; CX: non-shocked exposure to the chambers). (B) All illustrations show the mean and SEM levels of freezing. All rats decreased their freezing responses across extinction. (C) mPFC infusion of ifenprodil impaired the decrease of fear responses across re-extinction (Group IFEN/VEH) whereas muscimol depressed the fear responses (Group MUSC/VEH). (D) The retention test showed that ifenprodil impaired both acquisition and consolidation of re-extinction (Groups IFEN/VEH and VEH/IFEN). mPFC infusion of muscimol disrupted long-term loss of fear whether it was given before or after re-extinction (Groups MUSC/VEH and VEH/MUSC).

The test data on day 5 (Fig. 5D) revealed that NMDAr activation in the $\mathrm{mPFC}$ is necessary to consolidate relearning extinction of fear responses. Rats infused with ifenprodil after reextinction (Group VEH/IFEN) froze significantly more than vehicle-treated rats (Group VEH/VEH; $F_{(1,44)}=17.734, P<0.05$ ). Ifenprodil also impaired the long-term inhibition of fear responses when given prior to re-extinction. Rats that had been infused with ifenprodil (Group IFEN/VEH) froze significantly more than control rats (Group VEH/VEH; $F_{(1,44)}=22.379$, $P<0.05)$. Consistent with this role for NMDAr activation, disruption of neuronal activity in the $\mathrm{MPFC}$ impaired relearning. 
Rats that had received mPFC infusion of muscimol either before (Group MUSC/VEH) or after (Group VEH/MUSC) the second extinction session displayed significantly more freezing than control rats on the subsequent retention test (Group VEH/VEH; respectively: $F_{(1,41)}=16.386, P<0.05$ and $F_{(1,41)}=12.855$, $P<0.05)$.

\section{Discussion}

The present series of experiments studied the roles of the basolateral amygdala (BLA) and the medial prefrontal cortex (mPFC) in learning and relearning to inhibit fear responses in extinction. In Experiments 1 and 2, rats received context conditioning on days 1 and 3 followed by extinction on day 4 . In Experiments 3 and 4 , rats received context conditioning on day 1 , extinction on day 2 , reconditioning on day 3 , and re-extinction on day 4 . Finally, in each experiment, rats were tested on day 5 for retention of the inhibition produced by extinction or re-extinction.

Experiment 1 confirmed that blockade of NMDAr containing the NR2B subunit in the BLA disrupts the inhibitory learning produced by extinction (Sotres-Bayon et al. 2007; Laurent et al. 2008). Rats that received extinction under an infusion of the selective NMDAr antagonist ifenprodil took longer to inhibit fear responses across the session than control rats. Moreover, the ifenprodil-treated rats exhibited more fear responses than controls when subsequently tested drug-free. Thus, ifenprodil impaired both short- and long-term inhibition of fear responses, suggesting that NMDAr-mediated plasticity within the BLA mediates the development of inhibitory control over conditioned fear responses in extinction. In contrast to these effects on the development of inhibition, post-extinction manipulation of NMDAr activity spared long-term inhibition of fear responses. When ifenprodil was infused into the BLA immediately after extinction, rats showed low levels of fear responses similar to those of control rats on the subsequent retention test. These findings suggest that NMDAr containing the NR2B subunit in the BLA are involved in acquisition (Sotres-Bayon et al. 2007; Laurent et al. 2008) but not consolidation of the inhibitory learning produced by extinction. Experiment 1 also demonstrated that inactivation of the BLA prior to extinction impaired long-term inhibition of fear (Kim and Richardson 2008; Laurent et al. 2008). Rats infused in the BLA with muscimol prior to extinction froze significantly less than controls across the session, but substantially more when tested the following day. The depression of fear responding across the extinction session by muscimol is consistent with the view that activity in the BLA is crucial for the expression of the learning produced by conditioning (Helmstetter and Bellgowan 1994; Wilensky et al. 2006). Finally, Experiment 1 showed that inactivation of the BLA by muscimol also impaired long-term inhibition of fear when given after extinction. The impairment on the retention test among rats that received a pre- or postextinction infusion of muscimol shows that neuronal activity in the BLA is required for consolidation and suggests that it may also be required for acquisition of inhibition. Further, consolidation of inhibition involves mechanisms in the BLA other than activation of NMDAr containing the NR2B subunit (Lin et al. 2003; Herry and Mons 2004).

Experiment 1 demonstrated that activation of NMDAr containing the NR2B subunit in the BLA is critical for the development, but not the consolidation of the inhibitory learning produced by extinction. Experiment 2 provided evidence that activation of these receptors in the MPFC is critical for consolidation, but not development of this inhibition (Burgos-Robles et al. 2007). Infusion of ifenprodil into the mPFC did not impair the inhibition of fear responses across extinction but did impair retention of this inhibition when rats were subsequently tested drug free. Retention was also impaired when ifenprodil was infused into the MPFC after extinction, showing that activation of NMDAr containing the NR2B subunit is critical for consolidation (Burgos-Robles et al. 2007). Experiment 2 also demonstrated that infusion of muscimol before extinction reduced fear responses. This reduction is in line with previous evidence that activity in the mPFC, particularly the prelimbic area, contributes to expression of conditioned fear responses (Milad et al. 2004; SierraMercado Jr. et al. 2006; Vidal-Gonzalez et al. 2006). Importantly, pre-extinction infusion of muscimol also impaired long-term inhibition of fear responses. This impairment is likely to reflect a disruption of consolidation rather than learning. Pharmacological manipulations restricted to the mPFC typically lead to normal inhibition of fear responses across extinction but impaired retention of this inhibition the following day (Santini et al. 2004; Hugues et al. 2006; Burgos-Robles et al. 2007). Further, electrophysiological studies have shown that activity of mPFC neurons remains unchanged across extinction (Herry and Garcia 2002; Milad and Quirk 2002; Santini et al. 2008). Consistent with this role for the mPFC in consolidation but not learning, inactivation of the mPFC after extinction by an infusion of muscimol also impaired retention of inhibition. This last finding contrasts with a previous failure to detect an impairment of retention following post-extinction inactivation of the mPFC (Sierra-Mercado Jr. et al. 2006). The basis for this discrepancy is unclear but one possibility is that the type of CS used, a discrete auditory CS (SierraMercado Jr. et al. 2006) versus a continuous context CS in the present experiments, may determine the contrasting roles of the mPFC in consolidation of the inhibitory memory.

Experiment 3 confirmed that relearning extinction also involves NMDAr-mediated changes in plasticity within the BLA (Laurent et al. 2008). Rats that received their second extinction under ifenprodil took longer to inhibit fear responses than controls and exhibited substantial levels of fear responses on the subsequent retention test. Moreover, just as ifenprodil failed to affect consolidation after the initial extinction, the drug also failed to affect consolidation after the second. Whereas consolidation of the initial inhibition requires neuronal activity in the BLA (Experiment 1), relearning inhibition occurs when the BLA is temporarily inactivated (Kim and Richardson 2008; Laurent et al. 2008). Experiment 3 replicated these findings concerning the role of the BLA in learning and relearning inhibition. Infusion of muscimol in the BLA depressed fear responses on the second extinction as it had depressed those reactions on the first. However, infusion of muscimol before or after the second extinction did not produce the impairment observed when the drug had been infused before or after the initial extinction. In Experiment 3 , muscimol-treated rats displayed low levels of freezing similar to those shown by control rats on the subsequent retention test. Thus, these results reveal a paradox whereby blockade of NMDArmediated plasticity in the BLA disrupts relearning inhibition whereas disruption of both this plasticity and neural activity in the BLA spares relearning (Laurent et al. 2008).

Experiment 4 demonstrated that consolidation of relearning requires neuronal activity in the mPFC. Muscimol-induced inactivation of the mPFC before or after the second extinction session impaired the long-term inhibition of fear responses. Furthermore, infusion of the drug before re-extinction reduced fear responses as it had reduced these responses across initial extinction. This again indicates the critical role played by the mPFC in the expression of both conditioned and reconditioned fear responses (Milad et al. 2004; Sierra-Mercado Jr. et al. 2006; VidalGonzalez et al. 2006). Experiment 4 also demonstrated that the mechanisms involved in consolidation of relearning include NMDAr activation. Rats infused with ifenprodil after reextinction had learned to inhibit fear responses across the re- 
extinction session but did not retain this inhibition: They froze more than control rats on the subsequent retention test. The selective NMDAr antagonist also impaired long-term inhibition when administered before re-extinction. In addition, it impaired the inhibition of fear responses across that re-extinction session. This impairment in the inhibition of fear responses across reextinction contrasts with the absence of such an impairment across the initial extinction. This contrast in the effect of ifenprodil suggests that the substrate for the inhibition of fear responding shifts from extinction to re-extinction: Activation of NMDAr containing the NR2B subunit in the mPFC is not required for the development of inhibition across the initial extinction but is required for the development of inhibition across the second extinction. Alternatively, NMDAr activation in the mPFC may be critical for retrieval of the inhibitory memory formed at the initial extinction. Single-unit recording studies have demonstrated that presentation of an extinguished CS is accompanied by increased firing of mPFC neurons, implying that these neurons are involved in the retrieval of extinction memory (Herry and Garcia 2002; Milad and Quirk 2002; Santini et al. 2008). Moreover, as noted previously, reconditioning spares signature phenomena of extinction such as spontaneous recovery. This shows that the inhibitory memory formed by extinction survives reconditioning and is available for retrieval at the second extinction (Rescorla 2001; Leung and Westbrook, in press). Therefore, infusion of ifenprodil into the mPFC before reextinction may have impaired retrieval of the inhibitory memory formed at the initial extinction. Additional research is required to examine this possibility. For instance, if retrieval of the inhibitory memory requires activation of NMDAr containing the NR2B subunit, then infusion of ifenprodil into the $\mathrm{mPFC}$ would restore fear responses to an extinguished CS.

Contemporary models of the learning produced by extinction emphasize interactions among several brain regions, most notably the amygdala and the mPFC (Likhtik et al. 2005; Quirk and Mueller 2008). The results of Experiments 1 and 2 are consistent with such models. These results demonstrated that the BLA is critical for both the acquisition and consolidation of the learning that underlies the inhibition of fear responses in extinction. More specifically, they showed that activation of NMDAr containing the NR2B subunit mediates the plasticity underlying this learning (Sotres-Bayon et al. 2007; Laurent et al. 2008) but that some other processes in the BLA are involved in the longterm stabilization of the changes in synaptic efficiency (Lin et al. 2003; Herry and Mons 2004). Activation of these receptors in the mPFC does not mediate the development of learning but is required for the stabilization that underlies the long-term inhibition of fear responses (Burgos-Robles et al. 2007). Thus, the learning produced by extinction requires activation of these receptors in the BLA to produce changes in synaptic efficiency while the maintenance of these changes requires their activation in the mPFC. The results of Experiments 3 and 4 show that there are changes in the manner in which the BLA and MPFC interact when the extinguished context CS is subjected to an additional cycle of conditioning and extinction. Specifically, activation of NMDAr containing the NR2B subunit in the BLA remains critical for relearning to inhibit fear responses (Laurent et al. 2008) but the consolidation of this inhibition no longer requires neuronal activity in the BLA. Moreover, activation of these receptors in the mPFC remains critical for consolidation of this inhibition but their activation is now critical for relearning that inhibition. Thus, relearning to inhibit fear responses requires activation of NMDAr not only in the BLA but also in the mPFC to produce changes in synaptic efficiency while the maintenance of these changes again requires activation of these receptors in the mPFC.

The role of NMDAr activation in the BLA in mediating the learning produced by extinction is supported by the demonstration that inactivation of the BLA disrupts this learning. However, the role of NMDAr activation in the BLA in mediating the learning produced by re-extinction is challenged by the demonstration that inactivation of the BLA fails to disrupt this relearning. If NMDAr activation in the BLA is critical for relearning to inhibit fear responses, how can relearning occur when the BLA is inactivated? We suggest that the critical factor is the neuronal activity in the BLA that mediates the fear responses produced by reconditioning. This activity in the BLA requires the use of NMDAr in the BLA to relearn the inhibition that suppresses fear responses across re-extinction. Inactivation of neuronal activity in the BLA means that the subjects do not have to relearn to inhibit these fear responses across the second extinction session as no such responses are exhibited. Nevertheless, that session entails subjects being again exposed to the context CS in the absence of shock. We propose that this exposure retrieves and strengthens the inhibitory memory established by the initial extinction, and that this retrieval and strengthening involves activation of NMDAr in the mPFC. This proposal is entirely consistent with current neural models of fear extinction (Likhtik et al. 2005; Quirk and Mueller 2008). These models assume that, during extinction, the mPFC activates inhibitory mechanisms in the CeA that suppress fear responses to the extinguished CS. This inhibition counteracts the activation of the CeA by the BLA via projections of the MPFC to a network of inhibitory interneurons located between the BLA and the CeA (Pare and Smith 1993; McDonald 1998; Royer et al. 1999). This implies that the mPFC is able to re-exert its inhibitory control over the CeA when the BLA is inactivated across subsequent extinction.

\section{Materials and Methods}

\section{Subjects}

Subjects were 201 experimentally naïve male Wistar rats (Rattus norvegicus). They were obtained from a local supplier (Gore Hill Research Laboratories, Sydney, New South Wales, Australia) and weighed between 280 and $350 \mathrm{~g}$. They were housed in plastic boxes $(22 \mathrm{~cm}$ high $\times 65 \mathrm{~cm}$ long $\times 40 \mathrm{~cm}$ wide) located in a climate-controlled colony room under natural lighting. There were eight rats per box with food and water continuously available. Three days after arrival in the laboratory, rats were handled each day for $4 \mathrm{~d}$. The experimental procedures were approved by the Animal Ethics Committee at the University of New South Wales and conducted in accordance with the National Institutes of Health "Guidelines for the Care and Use of Laboratory Animals" (publication DHHS NIH 86-23). All experiments occurred between 7:00 A.M. and 10:00 P.M.

\section{Drugs}

The $\mathrm{GABA}_{\mathrm{A}}$ agonist, muscimol (Sigma), was dissolved in $0.9 \%$ $(\mathrm{wt} / \mathrm{vol})$ non-pyrogenic saline to obtain a final concentration of either $0.3 \mu \mathrm{g} / 0.3 \mu \mathrm{L}$ (infusion in the BLA) or $0.25 \mu \mathrm{g} / 0.5 \mu \mathrm{L}$ (infusion in the mPFC). Non-pyrogenic saline was used to control for any effect of the infusion procedures per se. Ifenprodil, a selective antagonist of NMDAr containing the NR2B subunit (Sigma), was dissolved in a solution of $0.9 \%$ non-pyrogenic saline (wt/vol) containing $5 \%$ of (2-hydrocypropryl)- $\beta$-cyclodextrin (Sigma) adjusted to pH 7.0. Non-pyrogenic saline containing 5\% of (2-hydrocypropryl)- $\beta$-cyclodextrin was used to control for any effect of the infusion procedures per se. Ifenprodil was microinjected at a final concentration of either $1 \mu \mathrm{g} / 0.3 \mu \mathrm{L}$ (infusions in the BLA) or $2 \mu \mathrm{g} / 0.5 \mu \mathrm{L}$ (infusions in the mPFC). All the doses were selected on the basis of previous investigations conducted in our laboratory (Laurent et al. 2008) or in the literature (Maren et al. 2001; Akirav et al. 2006; Sotres-Bayon et al. 2007).

\section{Surgery and drug infusion}

Rats received intraperitoneally (i.p.) injections of $1.3 \mathrm{~mL} / \mathrm{kg}$ of the anesthetic ketamine (Ketapex; Apex Laboratories) at a con- 
centration of $100 \mathrm{mg} / \mathrm{mL}$ and of $0.3 \mathrm{~mL} / \mathrm{kg}$ of the muscle relaxant xylazine (Rompun; Bayer) at a concentration of $20 \mathrm{mg} / \mathrm{mL}$. Anaesthetized rats were then mounted on a stereotaxic apparatus (Kopf Instruments), and 26 gauge guide cannulas (Plastics One) were implanted through holes drilled in the skull in one of the two targeted structures: the BLA (anteroposterior $[\mathrm{AP}]:-2.3 \mathrm{~mm}$; mediolateral $[\mathrm{ML}]: \pm 5 \mathrm{~mm}$; dorsoventral [DV]: -7.7$)$ or the mPFC (AP: $+2.9 \mathrm{~mm}$; ML: $+1 \mathrm{~mm}$; DV: $-4.1 \mathrm{~mm}$; angled $11^{\circ}$ toward the midline in the coronal plate). The guide cannulas were maintained in position with dental cement and dummy cannulas were kept in each guide at all times except during microinjections. Immediately after the surgical procedure, rats were injected i.p. with a prophylactic $(0.3 \mathrm{~mL})$ dose of $300 \mathrm{mg} / \mathrm{kg}$ solution of procaine penicillin. Rats were allowed $4 \mathrm{~d}$ to recover from surgery, during which time they were handled and weighed daily.

Ifenprodil, muscimol, and the appropriate vehicle were infused in either the BLA or the MPFC by inserting a 33 gauge internal cannula into the guide cannula. The internal cannula was connected to a $25-\mu \mathrm{L}$ glass syringe attached to an infusion pump (Harvard Apparatus) and projected an additional $1 \mathrm{~mm}$ ventral to the tip of the guide cannula. A total volume of either $0.3 \mu \mathrm{L}$ (infusion in the BLA) or $0.5 \mu \mathrm{L}$ (infusion in the mPFC) was delivered at a rate of $0.1 \mu \mathrm{L} / \mathrm{min}$. The internal cannula remained in place for a further $1 \mathrm{~min}$ after the infusions and was then removed. On the day before infusions, the dummy cannula was removed and the infusion pump was turned on for $3 \mathrm{~min}$ in order to familiarize the rats with the procedure and thereby minimize any stress produced by this procedure when infusions occurred.

\section{Histology}

Subsequent to behavioral testing, subjects implanted with cannulas received a lethal dose of sodium pentobarbital and their brains were removed and frozen. Unfixed brains were sectioned coronally at $40 \mu \mathrm{m}$ through the BLA or the mPFC. Every third section was collected on a slide, and the sections were stained with cresyl violet. The location of cannulas tips was determined under a microscope by a trained observer who was unaware of the subjects' group designations using the boundaries defined by Paxinos and Watson atlas (Paxinos and Watson 2007). Subjects with inaccurate cannula placements or with extensive damage to the BLA or the MPFC were excluded from the statistical analysis.

\section{Apparatus}

Four chambers, each measuring $20 \mathrm{~cm}$ height $\times 21 \mathrm{~cm}$ length $\times 23 \mathrm{~cm}$ width, were used for pre-exposure, fear conditioning, extinction training, and testing. The front and rear walls of these chambers, as well as the hinged lid, were constructed of Perspex, and the end walls were made of stainless steel. The floor in each chamber consisted of stainless steel rods, $2 \mathrm{~mm}$ in diameter, spaced $10 \mathrm{~mm}$ apart (center to center). The unconditioned stimulus (US) was a 1-sec, 0.8-mA unscrambled AC $50 \mathrm{~Hz}$ foot shock from a constant-current generator that was delivered to the floor of each chamber. The current available to each floor could be adjusted using an in-line milliampere meter. The floor of each chamber was $5 \mathrm{~cm}$ above a tray of paper pellet bedding (Fibrecycle) that was changed between rats. After removal of a rat, the floor of each chamber was cleaned with a solution of acetic acid $(1 \%)$ to eliminate any residue and provide a distinctive odor. The four chambers were located within separate compartments of a wooden cabinet. The door of each compartment was kept open to permit observation of the rat. The room that contained the experimental chambers was illuminated by four $60 \mathrm{~W}$ standard incandescent lights located in the ceiling. All experimental sessions were recorded on videotapes via a camera mounted on a wall opposite the chambers. The camera was connected to a monitor and video recorder located in an adjacent room.

\section{Behavioral procedures}

\section{Experiment 1: The role of the BLA in the learning produced by extinction}

This experiment examined the effects of infusing ifenprodil or muscimol in the BLA either before or after a first extinction ses- sion. To minimize any deficits in conditioned fear responses due to the surgical procedure, rats were exposed to the conditioning chambers for 2 min each day across four consecutive days. Such brief pre-exposures distributed across four days produce effects on subsequent conditioning that are the opposite to those produced by longer (e.g., $20 \mathrm{~min}$ ) exposures distributed across $14 \mathrm{~d}$. The brief pre-exposures used here facilitate the acquisition of context conditioned fear reactions, whereas longer and more frequent pre-exposures latently inhibit context conditioned fear responses (Kiernan and Westbrook 1993). All rats then received a conditioning episode on day 1 and day 3. Each episode consisted in placement of a rat in a chamber and delivering a foot shock $(0.8 \mathrm{~mA} ; 1 \mathrm{sec})$ one minute later. The rat remained in the chamber for a further two minutes before being returned to its home cage. On day 4, rats were infused in the BLA with vehicle (Groups VEH/VEH, VEH/IFEN, and VEH/MUSC), ifenprodil (Group IFEN/ $\mathrm{VEH}$ ), or muscimol (Group MUSC/VEH). Thirty minutes later, all rats received an extinction session which consisted in a 20-min exposure to the chambers in the absence of foot shock. Immediately after this session, all rats received a second infusion in the BLA. Some of the rats that had previously received vehicle now received ifenprodil (Group VEH/IFEN) or muscimol (Group VEH/ MUSC), whereas the rats that had been infused with ifenprodil or with muscimol now received vehicle (Groups IFEN/VEH and MUSC/VEH). Finally, control rats infused with vehicle before the extinction session also received vehicle after (Group VEH/VEH). Six hours later, all rats received counterbalanced infusions of drug or vehicle to control for any effect of ifenprodil or muscimol per se on subsequent test levels of freezing. Specifically, half of the control rats (Group VEH/VEH) were infused with ifenprodil while the remainder received muscimol. Rats in the remaining groups (Groups IFEN/VEH, VEH/IFEN, MUSC/VEH, and VEH/ MUSC) received an infusion of vehicle. Finally, all rats were tested drug-free across a 10-min non-shocked exposure to the chambers on day 5 .

\section{Experiment 2: The role of the mPFC in the learning produced by extinction}

This experiment examined the effects of infusing ifenprodil or muscimol in the mPFC either before or after an initial extinction session. Rats were pre-exposed to the context, and conditioned on days 1 and 3 as described in Experiment 1 . On day 4, rats were infused in the mPFC with vehicle (Groups VEH/VEH, VEH/IFEN, and VEH/MUSC), ifenprodil (Group IFEN/VEH), or muscimol (Group MUSC/VEH). Thirty minutes later, all rats received a 20 min extinction session to the context. Immediately after this session, all rats received a second infusion in the mPFC. Some of the rats that had previously received vehicle now received ifenprodil (Group VEH/IFEN) or muscimol (Group VEH/MUSC) whereas the remaining rats were infused with vehicle (Groups IFEN/VEH, MUSC/VEH, and VEH/VEH). Counterbalanced infusions were given to all rats six hours later as described previously. On day 5, all rats were tested in the manner described in Experiment 1 .

\section{Experiment 3: The role of the BLA in the learning produced by re-extinction}

This experiment examined the effects of infusing ifenprodil or muscimol in the BLA either before or after a second extinction session (i.e., re-extinction). It used the same procedure as in Experiment 1 except that an extinction session was inserted on day 2 . Thus, rats received two cycles of context conditioning and extinction: conditioning on day 1 and extinction on day 2 , reconditioning on day 3 and re-extinction on day 4 . The procedures used on the conditioning and extinction sessions were those described in Experiment 1. Thirty minutes before the reextinction session on day 4, rats were infused in the BLA with vehicle (Groups VEH/VEH, VEH/IFEN, and VEH/MUSC), ifenprodil (Group IFEN/VEH), or muscimol (Group MUSC/VEH). A second infusion occurred immediately after the session. Some of the rats that had previously received vehicle now received ifenprodil (Group VEH/IFEN) or muscimol (Group VEH/MUSC) 
whereas the remaining rats were infused with vehicle (Groups IFEN/VEH, MUSC/VEH, and VEH/VEH). Six hours later, all rats received counterbalanced infusions as described previously. Finally, all rats were tested on day 5 in the manner described.

\section{Experiment 4: The role of the mPFC in the learning produced by re-extinction}

This experiment examined the effects of infusing ifenprodil or muscimol in the mPFC either before or after a second extinction session (i.e., re-extinction). The procedure used in this experiment was similar to that used in Experiment 3. Rats received context conditioning on day 1 , extinction on day 2 , reconditioning to the context on day 3 , and re-extinction on day 4 . Thirty minutes before the re-extinction session, rats were infused in the mPFC with either vehicle (Groups VEH/VEH, VEH/IFEN, and VEH/MUSC) or ifenprodil (Group IFEN/VEH), or muscimol (Group MUSC/VEH). A second infusion occurred immediately after the session. Some of the rats that had previously received vehicle now received ifenprodil (Group VEH/IFEN) or muscimol (Group VEH/MUSC) whereas the remaining rats were infused with vehicle (Groups IFEN/VEH, MUSC/VEH, and VEH/VEH). Six hours later, all rats received counterbalanced infusions as described previously. Finally, all rats were tested on day 5 in the manner described.

\section{Scoring and statistics}

Freezing was defined as the absence of all movements, except those related to breathing (Blanchard and Blanchard 1969). The behavior of each rat was recorded on videotape, and freezing was rated with a time-sampling procedure in which each rat was observed every $2 \mathrm{sec}$ and scored as either freezing or moving. A percentage score was calculated for the proportion of the total observation that each rat spent freezing. Freezing was rated by two observers, one of whom was unaware of the subject's group designation. There was a high degree of agreement between the two observers: The Pearson product moment correlation between their rating was $>0.95$. The test data were analyzed in each experiment by a set of planned non-orthogonal contrasts that controlled the Family-Wise Error Rate using the Bonferroni inequality procedure (Harris 1994). Significance was set at the 0.05 level for each family of contrasts tested.

\section{Acknowledgments}

We thank Pankaj Sah and Nicola Watts for comments on an earlier version of this manuscript. This work was supported by a grant from the National Health and Medical Research Council of Australia.

\section{References}

Akirav, I., Raizel, H., and Maroun, M. 2006. Enhancement of conditioned fear extinction by infusion of the GABA agonist muscimol into the rat prefrontal cortex and amygdala. Eur. J. Neurosci. 23: 758-764.

Blanchard, R.J. and Blanchard, D.C. 1969. Crouching as an index of fear. J. Comp. Physiol. Psychol. 67: 370-375.

Bouton, M.E. and Swartzentruber, D. 1989. Slow reacquisition following acquisition: Context encoding and retrieval mechanisms. J. Exp. Psychol. Anim. Behav. Process. 15: 43-53.

Bouton, M.E., Westbrook, R.F., Corcoran, K.A., and Maren, S. 2006. Contextual and temporal modulation of extinction: Behavioral and biological mechanisms. Biol. Psychiatry 60: 352-360.

Burgos-Robles, A., Vidal-Gonzalez, I., Santini, E., and Quirk, G.J. 2007. Consolidation of fear extinction requires NMDA receptor-dependent bursting in the ventromedial prefrontal cortex. Neuron 53: 871-880.

Falls, W.A., Miserendino, M.J., and Davis, M. 1992. Extinction of fear-potentiated startle: Blockade by infusion of an NMDA antagonist into the amygdala. J. Neurosci. 12: 854-863.

Goosens, K.A. and Maren, S. 2004. NMDA receptors are essential for the acquisition, but not expression, of conditional fear and associative spike firing in the lateral amygdala. Eur. J. Neurosci. 20: 537-548.

Harris, R.J. 1994. ANOVA: An analysis of variance primer. Peacock, Itsac, IL.

Helmstetter, F.J. and Bellgowan, P.S. 1994. Effects of muscimol applied to the basolateral amygdala on acquisition and expression of contextual fear conditioning in rats. Behav. Neurosci. 108: $1005-1009$.

Herry, C. and Garcia, R. 2002. Prefrontal cortex long-term potentiation, but not long-term depression, is associated with the maintenance of extinction of learned fear in mice. J. Neurosci. 22: 577-583.

Herry, C. and Mons, N. 2004. Resistance to extinction is associated with impaired immediate early gene induction in medial prefrontal cortex and amygdala. Eur. J. Neurosci. 20: 781-790.

Herry, C., Trifilieff, P., Micheau, J., Luthi, A., and Mons, N. 2006 Extinction of auditory fear conditioning requires MAPK/ERK activation in the basolateral amygdala. Eur. J. Neurosci. 24: 261-269.

Hugues, S., Chessel, A., Lena, I., Marsault, R., and Garcia, R. 2006. Prefrontal infusion of PD098059 immediately after fear extinction training blocks extinction-associated prefrontal synaptic plasticity and decreases prefrontal ERK2 phosphorylation. Synapse 60: $280-287$.

Kiernan, M.J. and Westbrook, R.F. 1993. Effects of exposure to a to-be-shocked environment upon the rat's freezing response: Evidence for facilitation, latent inhibition, and perceptual learning. Q. J. Exp. Psychol. B 46: 271-288.

Kim, J.H. and Richardson, R. 2008. The effect of temporary amygdala inactivation on extinction and reextinction of fear in the developing rat: Unlearning as a potential mechanism for extinction early in development. J. Neurosci. 28: 1282-1290.

Kim, J., Lee, S., Park, H., Song, B., Hong, I., Geum, D., Shin, K., and Choi, S. 2007. Blockade of amygdala metabotropic glutamate receptor subtype 1 impairs fear extinction. Biochem. Biophys. Res. Commun. 355: 188-193.

Laurent, V., Marchand, A.R., and Westbrook, R.F. 2008. The basolateral amygdala is necessary for learning but not relearning extinction of context conditioned fear. Learn. Mem. 15: 304-314.

Lee, H. and Kim, J.J. 1998. Amygdalar NMDA receptors are critical for new fear learning in previously fear-conditioned rats. J. Neurosci. 18: $8444-8454$.

Leung, H.T. and Westbrook, R.F. Spontaneous recovery of extiguished fear responses deepens their extinction: A role for error-correction mechanisms. J. Exp. Psychol. Anim. Behav. Process. (in press).

Likhtik, E., Pelletier, J.G., Paz, R., and Pare, D. 2005. Prefrontal control of the amygdala. J. Neurosci. 25: 7429-7437.

Lin, C.H., Yeh, S.H., Lu, H.Y., and Gean, P.W. 2003. The similarities and diversities of signal pathways leading to consolidation of conditioning and consolidation of extinction of fear memory. $J$. Neurosci. 23: 8310-8317.

Maren, S., Yap, S.A., and Goosens, K.A. 2001. The amygdala is essential for the development of neuronal plasticity in the medial geniculate nucleus during auditory fear conditioning in rats. J. Neurosci. 21: RC135:1-6.

McDonald, A.J. 1998. Cortical pathways to the mammalian amygdala. Prog. Neurobiol. 55: 257-332.

Milad, M.R. and Quirk, G.J. 2002. Neurons in medial prefrontal cortex signal memory for fear extinction. Nature 420: 70-74.

Milad, M.R., Vidal-Gonzalez, I., and Quirk, G.J. 2004. Electrical stimulation of medial prefrontal cortex reduces conditioned fear in a temporally specific manner. Behav. Neurosci. 118: 389-394.

Pare, D. and Smith, Y. 1993. The intercalated cell masses project to the central and medial nuclei of the amygdala in cats. Neuroscience 57: 1077-1090.

Paxinos, G. and Watson, C. 2007. The rat brain in stereotaxic coordinates. Academic, Sydney, Australia.

Quirk, G.J. and Mueller, D. 2008. Neural mechanisms of extinction learning and retrieval. Neuropsychopharmacology 33: 56-72.

Quirk, G.J., Likhtik, E., Pelletier, J.G., and Pare, D. 2003. Stimulation of medial prefrontal cortex decreases the responsiveness of central amygdala output neurons. J. Neurosci. 23: 8800-8807.

Rescorla, R.A. 2001. Retraining of extinguished Pavlovian stimuli. J. Exp. Psychol. Anim. Behav. Process. 27: 115-124.

Rescorla, R.A. and Wagner, A.R. 1972. A theory of Pavlovian conditioning: Variations in the effectiveness of reinforcement and nonreinforcement. Appleton Century Crofts, New York.

Royer, S., Martina, M., and Pare, D. 1999. An inhibitory interface gates impulse traffic between the input and output stations of the amygdala. J. Neurosci. 19: 10575-10583.

Rumelhart, D.E. and McClelland, J.L. 1986. Parallel distributed processing: Explorations in the microstructure of cognition, Vol. 1. MIT Press, Cambridge, MA.

Santini, E., Muller, R.U., and Quirk, G.J. 2001. Consolidation of extinction learning involves transfer from NMDA-independent to NMDA-dependent memory. J. Neurosci. 21: 9009-9017.

Santini, E., Ge, H., Ren, K., Pena de Ortiz, S., and Quirk, G.J. 2004. Consolidation of fear extinction requires protein synthesis in the medial prefrontal cortex. J. Neurosci. 24: 5704-5710.

Santini, E., Quirk, G.J., and Porter, J.T. 2008. Fear conditioning and 
extinction differentially modify the intrinsic excitability of infralimbic neurons. J. Neurosci. 28: 4028-4036.

Sierra-Mercado Jr., D., Corcoran, K.A., Lebron-Milad, K., and Quirk, G.J. 2006. Inactivation of the ventromedial prefrontal cortex reduces expression of conditioned fear and impairs subsequent recall of extinction. Eur. J. Neurosci. 24: 1751-1758.

Sotres-Bayon, F., Bush, D.E., and LeDoux, J.E. 2007. Acquisition of fear extinction requires activation of NR2B-containing NMDA receptors in the lateral amygdala. Neuropsychopharmacology 32: 1929-1940.

Vidal-Gonzalez, I., Vidal-Gonzalez, B., Rauch, S.L., and Quirk, G.J. 2006. Microstimulation reveals opposing influences of prelimbic and infralimbic cortex on the expression of conditioned fear. Learn. Mem. 13: 728-733.

Wilensky, A.E., Schafe, G.E., Kristensen, M.P., and LeDoux, J.E. 2006 Rethinking the fear circuit: The central nucleus of the amygdala is required for the acquisition, consolidation, and expression of Pavlovian fear conditioning. J. Neurosci. 26: 12387-12396.

Received May 24, 2008; accepted in revised form July 16, 2008. 


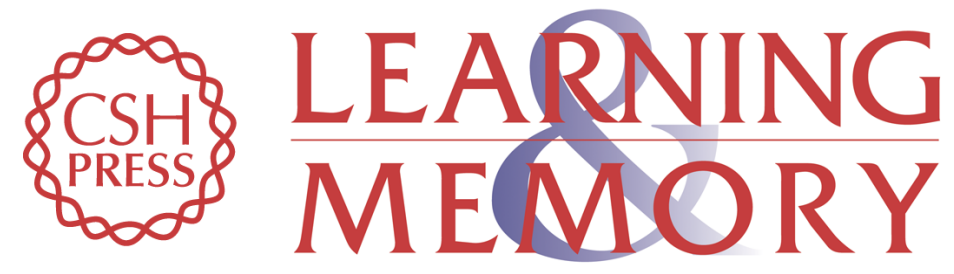

\section{Distinct contributions of the basolateral amygdala and the medial prefrontal cortex to learning and relearning extinction of context conditioned fear}

Vincent Laurent and R. Frederick Westbrook

Learn. Mem. 2008, 15:

Access the most recent version at doi:10.1101/lm.1080108

References This article cites 35 articles, 14 of which can be accessed free at: http://learnmem.cshlp.org/content/15/9/657.full.html\#ref-list-1

License

Email Alerting

Receive free email alerts when new articles cite this article - sign up in the box at the Service top right corner of the article or click here. 